No. 1

\title{
MAGNETIC PHASES \\ AND GENERALIZED $t-J$ MODELS \\ IN DOPED MOTT-HUBBARD INSULATORS
}

\author{
A.M. Oleś \\ Institute of Physics, Jagiellonian University, Reymonta 4, 30-059 Kraków, Poland
}

AND J. ZAANEN

Lorentz Institute for Theoretical-Physics, Leiden University

P.O.B. 9506, 2300 RA Leiden, The Netherlands

We review some recent results obtained for the dynamics of a single hole and for the ground states at finite hole doping in $t-J$ model. Next, we address the role of orbital degeneracy in doped Mott-Hubbard insulators and show examples of effective strong coupling models which include the orbital degrees of freedom. These new $t^{-} J$ models have interesting phase diagrams, with the new magnetic phases stabilized by a competition between magnetic energy and excitonic excitations. It is argued that the doped holes always bind to the excitons and that the new phases identified on the mean-field level give rise to local distortions of the lattice. We conclude that realistic $t-J$ models derived from the electronic structure of particular compounds may be successfully applied for understanding both the observed magnetic ground states, and the results of photoemission experiments, as we have demonstrated recently for $\mathrm{NiO}$.

PACS numbers: 74.72.- $h, 64.60 .-\mathrm{i}, 64.90 .+\mathrm{b}, 75.40 .-\mathrm{s}$

\section{Hole doping in $t-J$ model}

Strongly correlated fermions, occur in nature in heavy-fermion systems, high temperature superconductors (HTS), doped Mott-Hubbard insulators, in ${ }^{3} \mathrm{He}$, and in neutron stars. Generally speaking, these systems involve orbitally degenerate states, as $3 d$ or $4 f(5 f)$ states. Yet, in the most common approach to describe the strongly correlated electrons the orbital degrees of freedom are ignored and the problem is reduced to that of spin degeneracy alone. While the nondegenerate Hubbard model, describing the electron correlations in the nondegenerate $s$ band has been used with some success to describe the physical properties of ${ }^{3} \mathrm{He}$ [1], the attempts to classify various magnetic, charge order, and superconducting instabilities observed in solid state physics in terms of electrons interacting within 
a nondegenerate band [2] may serve only for qualitative, but not for quantitative understanding. This is particularly obvious in heavy fermion systems where it is generally believed that many of the unexplained features are related to orbital degeneracy. Despite the existing counterevidence, in the absence of thorough understanding of the role played by orbital degeneracy it might be premature to discard it completely in the context of high $T_{\mathrm{c}}$ superconductivity [3] and concentrate just on the properties which follow either from the nondegenerate Hubbard model [4], or from the so-called three-band model which deals with $\mathrm{Cu}\left(3 d_{x^{2}-y^{2}}\right)$ and $\mathrm{O}\left(2 p_{\sigma}\right)$ orbitals, one orbital per each site [5]. But so far, with few exceptions, like the $1 / N$-expansion for Ce intermetallics [6], the quadrupolar Kondo effect [7], or the study of magnetic phases in degenerate $e_{\mathrm{g}}$ band [8], orbital degeneracy is not considered. We will argue below that in certain (doped) Mott-Hubbard insulators the orbital degrees of freedom do play an important role and the physics is different when they are discarded from the start.

The essential property of strongly correlated systems is a dramatic reduction of certain charge fluctuations, compared to those of independent electron case. In the extreme case this is expressed mathematically by taking the limit of infinite Coulomb interaction, $U \rightarrow \infty$, as frequently done in the theory of heavy-fermion systems [9]. If this is done in the half-filled Hubbard model, the fermions are frozen and the ground state has $2^{N}$ degeneracy with respect to their spin orientations. Doped holes can move only in the projected space, without producing doubly occupied configurations. The problem is still far from trivial and the only exact solution (apart from one dimension) is the ferromagnetic (FM) polaron obtained by Nagaoka in 1966 [10]. It demonstrates that doping of this (infinitely) strongly correlated system removes the spin degeneracy and promotes FM order, in fact stable up to the concentration of holes of $\delta \approx 0.29$ in a cubic three-dimensional lattice [11]. In this extreme case a transition from one (spin-degenerate) to another (FM) ground state takes place at infinitesimally small concentration of holes due to the absence of any other magnetic interaction. It is this competition between the kinetic energy of the doped carriers and the magnetic order present in the system that we want to concentrate on in this short review. We will argue that such a competition is common for all Mott-Hubbard insulators and show on a few examples that doping leads in these systems to the onset of new magnetic phases and, in certain regimes of parameters, to phase separation. The discussion will concentrate on the models realized in two dimensions on bipartite lattices, as these are relevant for the HTS and the related oxides.

We start with a strongly correlated nondegenerate band close to half-filling. At large (but finite) Coulomb interaction $U$ the ground state of the system consists of singly-occupied (spins) and empty (holes) configurations, while the double occupancies can be virtually excited and lead to an effective exchange interaction. In this limit, the Hubbard model can be replaced by an effective strong coupling model, the so-called $t-J$ model [12],

$$
H_{t J}=t \sum_{\langle i j\rangle \sigma}\left[\left(1-n_{i \sigma}\right) a_{i \sigma}^{\dagger} a_{j \sigma}\left(1-n_{j \sigma}\right)+\text { h.c. }\right]+J \sum_{\langle i j\rangle}\left(S_{i} \cdot S_{j}-\frac{1}{4} n_{i} n_{j}\right),
$$

where $J=4 t^{2} / U$ and the sums over $\langle i j\rangle$ include each pair of nearest neighbours only once. $a_{i \sigma}^{\dagger}$ is a creation operator for a hole with spin $\sigma$ at site $i$ and $S_{i}=$ 
$\left(S_{i}^{+}, S_{i}^{-}, S_{i}^{z}\right)$ is a spin operator at site $i$, related to the fermion (hole) operators in the usual way. The $t-J$ model does not include the three-site terms [12] which stand for the second nearest-neighbour effective hopping and contribute to the propagation of the holes [13]. Here we will concentrate on the properties of the $t-J$ model as defined in Eq. (1).

The discovery of high temperature superconductivity triggered a tremendous theoretical effort to understand the properties of the $t-J$ model after Anderson proposed that it could be considered as generic for the pairing mechanism in these materials [14]. Furthermore, the $t-J$ model was derived from a realistic three-band model which describes the electronic structure of the HTS superconductors [15]. In spite of this effort, the $t-J$ model has been solved exactly only for $J=2 t$ in one dimension [16] and we have to rely on approximate solutions. At half-filling the $t-J$ model is equivalent to a Heisenberg antiferromagnet and one finds an antiferromagnetic (AF) ground state with long-range order (LRO) in two dimensions [17].

Before discussing the phase diagram at finite doping, let us first discuss some significant progress in the understanding of the carrier propagation in the Mott-Hubbard insulators as achieved recently in the studies of a single hole doped in an antiferromagnet, described by the $t-J$ model (1). It is well known that the hole spectral function in the AF background is totally incoherent in the limit of $J \rightarrow \infty$, as shown in the nonretractable path approximation [18]. However, the ground state is in this case FM, as the kinetic energy of a single hole dominates the problem [10]. At finite $J$ the motion of an added hole is hindered on the mean-field level by the strings of reversed AF bonds which increase the magnetic energy proportionally to the length of the hole path [19]. Therefore, the coherent propagation may occur only due to processes involving closed loops, in which a moving hole repairs the defects in the spin order by retracing its own path. The simplest of those involves six elementary hoppings on a square lattice [20]. The situation changes drastically when the quantum fluctuations are included from the start, as they are able to repair the defects in the magnetic structure which appear on the string [21]. As a result, a coherent quasiparticle mode appears at low energies, with the dispersion $\sim J[13,22]$, consistent with the overlap of the hole wave functions dressed by local quantum fluctuations [23]. This problem may be represented as a scattering of holes with the creation (annihilation) of a single spin wave in a single scattering event. Fortunately, the vertex corrections decrease with the increasing value of $J$, while the $Q P$ mode gradually dominates the spectrum, and the spectral function calculated in self-consistent Born order agrees quite well with the results of numerical calculations for finite clusters [24].

Having in mind the results obtained for one hole, one may expect that the quantum fluctuations are crucial to understand quantitatively the transition from the Mott insulating AF state at half-filling to other magnetic states, including a strongly correlated metal, with increasing doping. Indeed, the problem of the phase diagram of the $t-J$ model as a function of doping proved to be very difficult, but certain qualitative statements can be made based on the results following from different kinds of mean-field approximations. All these studies indicate that incommensurate LRO is stabilized by doping, as the kinetic energy on the scale of $\sim t$ is allowed once the spins are not precisely antiparallel. Therefore, the system prefers a spiral or canted arrangement of the spins, rather than an AF one. While 
these two phases are equivalent classically, quantum fluctuations favour a spiral over canted structure [25]. The resulting pitch increases with doping and eventually the ground state becomes FM, i.e. the whole solid turns into a FM polaron. We can illustrate this gradual change of the magnetic ground state by using a simple mean-field theory formulated in terms of Schwinger bosons. The $t-J$ model is rewritten as [26],

$$
\begin{aligned}
H_{t J} & =t \sum_{\langle i j\rangle \sigma}\left(b_{j \sigma}^{\dagger} b_{i \sigma} f_{i}^{\dagger} f_{j}+\text { h.c. }\right) \\
& -\frac{1}{2} J \sum_{\langle i j\rangle \sigma \sigma^{\prime}} \sigma \sigma^{\prime}\left(1-f_{i}^{\dagger} f_{i}\right) b_{i \sigma^{\prime}}^{\dagger} b_{i \sigma^{\prime}} b_{j-\sigma^{\prime}}^{\dagger} b_{j-\sigma^{\prime}},
\end{aligned}
$$

where the original hole operators, $a_{i \sigma}^{\dagger}=b_{i \sigma} f_{i}^{\dagger}$, are replaced by Schwinger boson operators $b_{i \sigma}$ and by hole-fermion operators $f_{i}$. The ground state is approximated by the classical ansatz,

$$
\left|\Phi_{0}\right\rangle=\prod_{i}\left[\cos \theta_{i} b_{i \uparrow}^{\dagger}+\sin \theta_{i} b_{i \downarrow}^{\dagger}\right]|0\rangle,
$$

where the (uniform) difference between the two angles at nearest-neighbour sites, $\Theta=\left|\theta_{i}-\theta_{j}\right|$, equals $\pi / 2$ in the AF state at half-filling. As a result one finds the energy normalized per site

$$
E=-2 t \delta \cos \Theta-J(1-\delta)^{2} \sin ^{2} \Theta
$$

From the minimization of energy (4) over $\Theta$ one finds that the pitch increases as a function of doping $\delta$,

$$
\cos \Theta=\frac{t \delta}{J(1-\delta)^{2}} \text {. }
$$

Thus, the AF state is replaced by a spiral already at infinitesimal doping in this approximation. At higher doping the spiral approaches gradually the FM phase, reached at a critical concentration found from Eq. (5),

$$
\delta_{\mathrm{c}}=1+\frac{t}{2 J}-\sqrt{\frac{t}{2 J}\left(2+\frac{t}{2 J}\right)} .
$$

The numerically obtained phase diagram is presented in Fig. 1. The classical spin approximation (3) captures already the essence of the changes in the magnetic order induced by doping. First, the AF order is replaced by a spin spiral, which goes over into a FM solution at high doping. However, the classical ansatz (3) does not include correctly the energy gain due to quantum fluctuations in the AF and spiral phases. Thus, the region of stability of the AF phase with the largest quantum corrections is underestimated. A better mean-field slave boson approach was proposed by Jayaprakash et al. [26] who diagonalized the MF problems for bosons and fermions. The results are included for comparison in Fig. 1. The AF phase is stable at finite doping for small $t / J$. The stability of the spiral phase with respect to the FM one is also increased due to quantum fluctuations. In addition to the phases given in Fig. 1, the latter approximation also yields a disordered phase at high doping, $\delta>0.6$ and $t / J<0.2$. For the parameters relevant for HTS, $t \approx 0.4 \mathrm{eV}$ and $J \approx 0.13 \mathrm{eV}[27]$, both approximations nicely agree and predict the onset of spiral order at small doping. 


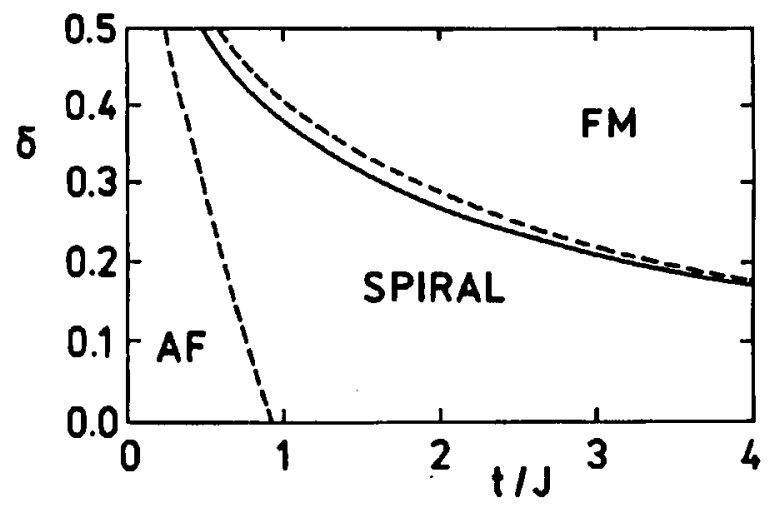

Fig. 1. Phase diagram of the $t-J$ model as obtained in the simple mean-field theory presented in the paper (full lines) and in the Schwinger boson mean-field of Jayaprakash et al. [26] (dashed lines).

Unfortunately, the above picture is strongly oversimplified, as the homogeneous magnetic states are locally unstable against the increase in the spiral pitch [28] and the $t-J$ model exhibits a strong tendency towards phase separation $[29,30]$. This tendency occurs as the holes are expelled from an AF state and can be easily understood in the regime of large $J$. Consider four holes doped to the half-filled $t-J$ model. On one hand, if they are in separate regions, the magnetic energy of $\Delta E=2 z J$ is lost in the Néel state, where $z$ is the number of nearest-neighbours. On the other hand, for a cluster of four holes on a square lattice one looses only the energy $\Delta E=(2+z) J$. At small $J$ the motion of a hole in an antiferromagnet is frustrated and FM polarons are formed [30]. Thus the system separates into hole-poor AF and hole-rich FM regimes. The instability towards phase separation exists thus for infinitesimal doping at any value of $J / t$ $[28,30]$. If the doping is increased, homogeneous solutions become stable again $[29,30]$. For instance, if $t / J=3$, as in HTS, the phase separation is expected in the $t-J$ model up to $\delta \approx 0.15$ [29].

The tendency towards phase separation is present not only in a strongly correlated nondegenerate band. It persists in the Hubbard model for weak and intermediate Coulomb interaction, and leads to the formation of incommensurate phases with domain walls [31]. Similar solutions are also found in a three-band model for the parameters realistic for HTS [28]. Indeed, experimental evidence is accumulating that phase separation occurs in HTS [32]. It follows from the weak dependence of the Fermi level on doping and the high hole concentrations required for superconductivity. Furthermore, the inhomogeneities are directly observed in the neutron scattering studies of the positions of apical oxygens. Such local inhomogeneities as charge or spin polarons, bipolarons, excitons, or domain walls are.in turn very susceptible to the changes of the electron-lattice interaction [33] and the electronic system cannot be considered in isolation from the lattice. There has been considerable progress in the understanding of the resulting ground states by finite size diagonalization and incommensurate Hartree-Fock studies of the three-band 
model [34]. We believe that these tendencies have to be particularly pronounced in the strongly correlated limit, where one deals with the effective Hamiltonians of the $t-J$ variety. However, this subject is still underdeveloped and we will limit our discussion to the electronic degrees of freedom.

\section{High spin $t-J$ model for $d^{9}$ configuration in $\mathrm{La}_{2} \mathrm{CuO}_{4}$}

If one wants to understand the magnetic ground states which occur under doping in a particular class of compounds, the $t-J$ model does not suffice and one has to consider holes interacting within particular orbitals. The aim is then to derive an effective model which deals only with the effective holes within the $d$ orbitals. We start with HTS, where it has been argued that the doping leads to the formation of Zhang-Rice bound states [15] and thus the problem may be reduced to the $t-J$ model. However, if one considers only the $\mathrm{Cu}(3 d)$ orbitals, and their respective crystal field splittings [35], the $\mathrm{Cu}\left(d_{3 z^{2}-1}\right)$ orbital is the first unoccupied orbital (by holes) in the undoped compound. The hole-hole interactions may be then written in the rotationally invariant form [36]:

$$
\begin{aligned}
& H_{\mathrm{int}}=\left(U+2 J_{H}\right) \sum_{i}\left(n_{i x \uparrow} n_{i x \downarrow}+n_{i z \uparrow} n_{i z \downarrow}\right)+\sum_{i \sigma}\left[U n_{i x \sigma} n_{i z-\sigma}\right. \\
& \left.+\left(U-J_{H}\right) n_{i x \sigma} n_{i z \sigma}-\frac{1}{2} J_{H} d_{i x, \sigma}^{\dagger} d_{i x,-\sigma} d_{i z,-\sigma}^{\dagger} d_{i z, \sigma}\right]+E_{z} \sum_{i \sigma}\left(n_{i z, \sigma}-n_{i x, \sigma}\right),(7)
\end{aligned}
$$

where the indices $x$ and $z$ stand for the orbitals $d_{x^{2}-y^{2}}$ and $d_{3 z^{2}-1}$, respectively. $U$ and $J_{H}$ stand for the Coulomb and exchange interactions. The last term in Eq. (7) is the crystal field energy parametrized by a single parameter $E_{z}$. The hopping part of the Hamiltonian is of the form

$$
H_{0} \sim t \sum_{\langle i j\rangle, \sigma}\left[d_{i x \sigma}^{\dagger} d_{j x \sigma}+\frac{1}{\sqrt{\alpha_{0}}}\left(d_{i x \sigma}^{\dagger} d_{j z \sigma}+\text { h.c. }\right)+\frac{1}{\alpha_{0}} d_{i z \sigma}^{\dagger} d_{j z \sigma}\right],
$$

with $\alpha_{0}=3$, if atomic orbitals are considered [37]. Doping in a system described by this Hamiltonian, $H=H_{0}+H_{\text {int }}$, leads to populating excitons, if only $E_{z}<J_{H}$, as it is the case for the realistic parameters [38]. There are some theoretical arguments which point out the importance of the $d_{3 z^{2}-1}$ orbitals [39], and it follows as well from the band structure that the value of the gap in HTS may be qualitatively understood only if the $a_{1}$ symmetry is explicitly included [40]. However, the recent experimental data seem to give only a small $(\approx 10 \%)$ occupancy of the $d_{3 z^{2}-1}$ orbital [5], in agreement with the analysis of the multiband model in the Hartree-Fock approximation with the configuration interaction corrections [38]. Thus, while the $a_{1}$ symmetry is certainly important in the HTS, the doping is not in the $3 d$ orbitals, but rather in the apical oxygen positions and the situation is even more complicated than we are describing here by the above model (7)-(8). Nevertheless, we shall consider this model as a starting point in this chapter to demonstrate how the competition between the magnetic interactions and the kinetic energy happens in such a situation.

At a filling of one hole per site, the hopping (8) is entirely suppressed for large $U$. One may then derive effective magnetic couplings between the localized holes by using perturbation theory. Although for one particular choice of 
the ground state the resulting interaction may be simpler, in general one finds a complicated Hamiltonian [41-43]:

$$
\begin{aligned}
H_{J} & =\sum_{\langle i \delta\rangle}\left[2 J_{i \delta}\left(\boldsymbol{S}_{i, x x}+\frac{1}{\alpha_{0}} \boldsymbol{S}_{i, z z}+\frac{(-1)^{y \cdot \delta}}{\sqrt{\alpha_{0}}} S_{i, x z}\right)\right. \\
& \times\left(S_{i+\delta, x x}+\frac{1}{\alpha_{0}} S_{i+\delta, z z}+\frac{(-1)^{y \cdot \delta}}{\sqrt{\alpha_{0}}} S_{i+\delta, x z}\right) \\
& \left.+\left(n_{i,-}+\frac{(-1)^{y \cdot \delta}}{\sqrt{\alpha_{0}}} T_{i, x z}\right)\left(n_{i+\delta,-}+\frac{(-1)^{y \cdot \delta}}{\sqrt{\alpha_{0}}} T_{i+\delta, x z}\right)\right] \\
& -\sum_{i}\left(E_{z}+\frac{z J}{\alpha_{0}^{2}}\right) n_{i,-.}
\end{aligned}
$$

In addition to the standard spin operators within a given orbital, $x\left(d_{x^{2}-y^{2}}\right)$ or $z$ $\left(d_{3 z^{2}-1}\right)$, the Hamiltonian depends as well on the interorbital spin operators

$$
\begin{aligned}
& S_{i, x z}^{+}=d_{i x \uparrow}^{\dagger} d_{i z \downarrow}+d_{i z \uparrow}^{\dagger} d_{i x \downarrow}, \\
& S_{i, x z}^{z}=\frac{1}{2}\left(d_{i x \uparrow}^{\dagger} d_{i z \uparrow}+d_{i z \uparrow}^{\dagger} d_{i x \uparrow}-d_{i x \downarrow}^{\dagger} d_{i z \downarrow}-d_{i z \downarrow}^{\dagger} d_{i x \downarrow}\right),
\end{aligned}
$$

which fulfil the standard commutation relations, and on spin-independent operators,

$$
\begin{aligned}
& n_{i,-}=\frac{1}{2}\left(d_{i x \uparrow}^{\dagger} d_{i x \uparrow}+d_{i x \downarrow}^{\dagger} d_{i x \downarrow}-d_{i z \uparrow}^{\dagger} d_{i z \uparrow}-d_{i z \downarrow}^{\dagger} d_{i z \downarrow}\right) \\
& T_{i, x z}=\frac{1}{2}\left(d_{i x \uparrow}^{\dagger} d_{i z \uparrow}+d_{i x \downarrow}^{\dagger} d_{i z \downarrow}+d_{i z \uparrow}^{\dagger} d_{i x \uparrow}+d_{i z \downarrow}^{\dagger} d_{i x \downarrow}\right)
\end{aligned}
$$

For simplicity, the terms which follow from the Hund rule exchange interaction $J_{H}$ [42] were not included in Eq. (9).

As we have discussed in more detail elsewhere [41, 42], the spin-orbital model (9) has a rather interesting phase diagram. If the Hund rule interaction $J_{H}$ is neglected, as in Eq. (9), the ground state is AF, with the holes occupying the $d_{x^{2}-y^{2}}\left(d_{3 z^{2}-1}\right)$ orbitals for $E_{z}>-16 J / 9\left(E_{z}<-16 J / 9\right)$. Finite $J_{H}$ promotes a FM phase near the transition point at $E_{z}=16 J / 9$, which exhibits an interesting two-sublattice orbital order [41, 42]. In fact, such a phase was found before in a similar (and less complete) Kugel-Khomskii model [44] and served as an explanation of the experimentally observed $\mathrm{FM}$ order in $\mathrm{K}_{2} \mathrm{CuF}_{4}$ [45]. It was one of the few successes of these complicated theories, where a theoretical prediction [44] was confirmed by the experiment [45].

Let us consider now doping by holes of the system described by the spin-orbital model at half-filling. Depending on the crystal field splitting, $E_{z}$, the doping may give either a triplet or a singlet local state. The singlet situation is equivalent to the usual $t-J$ model as discussed above. If a triplet is created, its hopping differs qualitatively from that of the singlet, as it possesses an internal spin degree of freedom which can be exchanged with the magnetic lattice. There are two factors which decide about the amplitude of the triplet hopping: (i) the character of the hopping hole ( $x$ or $z$ ), and (ii) the initial and final spin states with respect to the 
hopping process. Taking these aspects into account, one may derive the following Hamiltonian for the hopping of triplet holes [46]:

$$
\begin{aligned}
H_{t}= & \frac{t}{2} \sum_{i, \delta} \sum_{m, n=-1 / 2}^{1 / 2}\left\{\left[b_{i+\delta, z, n}^{\dagger}+\frac{(-1)^{\delta \cdot y}}{\sqrt{\alpha_{0}}} b_{i+\delta, x, n}^{\dagger}\right]\right. \\
& \times\left[\sum_{ \pm} \sqrt{\left(\frac{3}{2} \pm m\right)\left(\frac{3}{2} \pm n\right)} a_{i, m \pm 1 / 2}^{\dagger} a_{i+\delta, n \pm 1 / 2}\right] \\
& \left.\times\left[b_{i, z, n}+\frac{(-1)^{\delta \cdot y}}{\sqrt{\alpha_{0}}} b_{i, x, n}\right] h_{i} h_{i+\delta}^{\dagger}+\text { h.c. }\right\} .
\end{aligned}
$$

$b_{i x \sigma}^{\dagger}$ and $b_{i z \sigma}^{\dagger}$ are SU(2) Schwinger boson operators and have the same meaning as those introduced in Sec. 1. We used here the products of SU(3) Schwinger bosons, $a_{i m_{x}}^{\dagger}$, and auxiliary fermions, $h_{i}^{\dagger}$, to describe the internal spin degree of freedom $\left(m_{z}=1,0,-1\right)$ and the charge of the moving triplet hole, respectively [46]. The phase factors $(-1)^{\delta} \cdot \boldsymbol{y}$ come from the difference in the sign of the hoppings in the $x$ and $y$ directions ( $y$ is a vector pointing in the $y$ direction) which follows from the parity of the $d_{x^{2}-y^{2}}$ and $d_{3 z^{2}-1}$ orbitals. Equations (9) and (12) define a $t-J$ model for a triplet hole. The physics of this model is more complex than that of the singlet model. The first qualitative difference is visible by looking at the triplet hole moving in the Néel state. Due to the possibility of changing its own component of the spin, it can move freely in an AF lattice, without leaving behind a string of frustrated bonds. The coupling of such a hole to spin waves dresses this free propagation, but appears to be divergent at small momenta [46]. Thus, unlike in the singlet hole case, the vertex corrections are large and one has to go beyond the self-consistent Born order to solve the one-hole problem.

To simplify the situation, we refrain from discussing the complicated couplings of the magnetic and orbital degrees of freedom at finite doping and consider instead the nature of the structural instabilities occurring due to the coupling between the carriers and $d-d$ excitons. Disregarding the spin problem, let us reconsider the relatively simple spinless model $[42,43]$ :

$$
\begin{aligned}
H= & t \sum_{i, \delta}\left(b_{i z}+\frac{(-1)^{\delta} \cdot y}{\sqrt{\alpha_{0}}} b_{i x}\right) f_{i}^{\dagger} f_{i+\delta}\left(b_{i+\delta, z}^{\dagger}+\frac{(-1)^{\delta} \cdot y}{\sqrt{\alpha_{0}}} b_{i+\delta, x}^{\dagger}\right) \\
& +\sum_{i}\left[E_{z} b_{i z}^{\dagger} b_{i z}+\lambda_{i}\left(b_{i x}^{\dagger} b_{i x}+b_{i z}^{\dagger} b_{i z}+f_{i}^{\dagger} f_{i}-1\right)\right]
\end{aligned}
$$

which captures the essential features in the orbital channel. This model is motivated here by the independence of the orbital dynamics in the hopping Hamiltonian of the spin channels. We use slave-bosons, $b_{i x}^{\dagger}$ and $b_{i z}^{\dagger}$, to represent the orbital degrees of freedom, and enforce the usual local constraint at finite dopings by including Lagrange multipliers $\lambda_{i}$. The spinless model is solved in the slave-boson mean-field (MF) approximation, in which the $b_{i \xi}$ operators are replaced by scalars $\langle b\rangle$, and the local constraint $\left(\lambda_{i}\right)$ are replaced by a global one, i.e. $\lambda_{i}=\lambda$. The hole motion 
is solved with respect to the undoped ground state which represents the physical vacuum

$$
\left|\Phi_{0}\right\rangle=\prod_{i}\left[\alpha_{i x} b_{i x}^{\dagger}+\alpha_{i z} b_{i z}^{\dagger}\right]|0\rangle
$$

In its region of stability the mixed phase, composed of the $x$ and $z d^{9}$ local states, may have either uniform or staggered phasing. It can be verified that the lower free energy is obtained with the uniform phasing which gives,

$$
\mathcal{F}_{\mathrm{MF}}=\eta^{2} b^{2} E_{z}+\lambda\left[b^{2}\left(1+\eta^{2}\right)+\delta-1\right]-\frac{1}{\beta} \int \frac{\mathrm{d}^{2} k}{(2 \pi)^{2}} \ln \left[1+\exp \left(-\beta E_{k}\right)\right]
$$

where $\delta$ is the hole doping, $\beta=1 / k_{\mathrm{B}} T$, and $E_{k}$ are quasiparticle energies,

$$
E_{k}=\lambda+z t b^{2}\left[\left(\eta^{2}+\frac{1}{\alpha_{0}}\right) \gamma_{k}+\frac{2 \eta}{\sqrt{\alpha_{0}}} \gamma_{k}^{-}\right] .
$$

Here we use the boson mean-field amplitudes $\left\langle b_{x}\right\rangle=b$ and $\left\langle b_{z}\right\rangle=\eta b$, while $\gamma_{k}=\frac{1}{z} \sum_{\delta} \exp (-\mathrm{i} k \cdot \delta)$ and $\gamma_{\bar{k}}^{-}=\frac{1}{z} \sum_{\delta}(-1)^{y \cdot \delta} \exp (-\mathrm{i} \boldsymbol{k} \cdot \delta)$. As a result of numerical minimization of Eq. (15) one finds three saddle points. For $T \rightarrow 0$, the $d^{9}$ background is either of pure $d_{x^{2}-y^{2}}$ character (for large $E_{z}$ ), or of pure $d_{3 z^{2}-1}$ character (for small $E_{z}$ ), or, finally, a mixed state where both orbitals are partially occupied. In addition, near the transition from the $x$ to the mixed phase both uniform phases are unstable and one finds a region, where the holes phase separate into a hole-poor $x$ state and hole-rich mixed state, as shown in Fig. 2. Looking

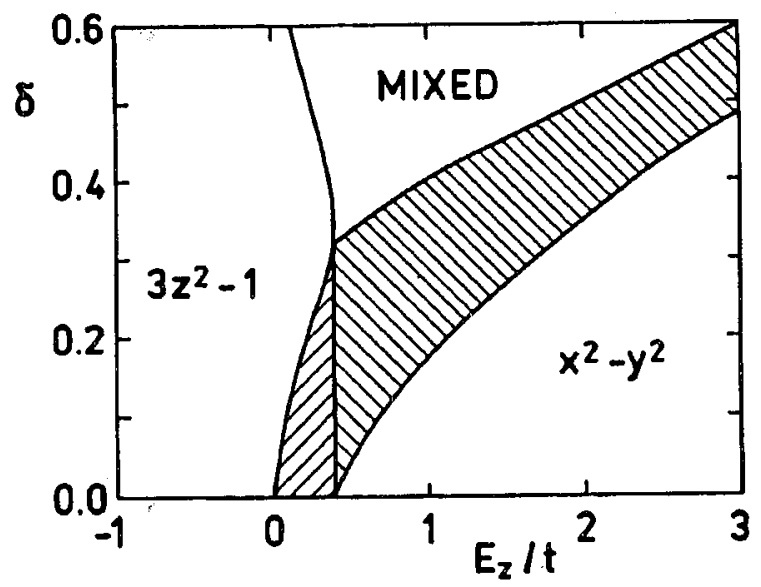

Fig. 2. Phase diagram of the spinless model (13) found in the mean-field approximation, as a function of the $d-d$ excitation energy, $E_{z} / t$, and hole concentration, $\delta$. The shaded areas indicate regions of phase separation between the pure and mixed phases.

at the result we note that the doping induces the phase separation and the new mixed phase (see Fig. 2). As in the case of the $t-J$ model, the phase separation is here a consequence of the existing competition between the crystal-field and the 
kinetic energy. More kinetic energy is gained, if an $x$-hole hops to a neighbouring site, but this means a preexisting excitonic excitation which costs the energy $E_{z}$. Of course, the energy is gained only if $E_{z}$ is not too large. Thus, the FM polaron of a singlet $t-J$ (or Hubbard) model is replaced here by an excitonic excitation which binds to the moving hole. This new kind of bound state gives rise to attractive interactions between holes which may be formally derived [42]. As a result, one finds a superconducting state promoted by the difference in the kinetic energy of the moving holes which couples to $d-d$ excitons. This new mechanism of pairing is of course different from the excitonic mechanisms related to the intersite Coulomb interactions [47], or the interaction between the electric quadrupolar moments [48].

It appears that the above picture is most probably too oversimplified to account for the physical reality. First of all, we neglected completely the magnetic degrees of freedom which were shown before to lead to a mixed phase already without doping [42]. In that case, however, the phasing of orbitals is staggered, therefore one may expect another competition between two different kinds of mixed phases under increasing doping. Furthermore, the uniform phasing is a direct consequence of the Hund rule exchange $J_{H}$ which is a fundamental interaction in the model, stabilizing the discussed triplet holes. Thus, it appears impossible to decouple the spin and orbital degrees of freedom. Furthermore, another complication comes from phonons. The staggered phasing couples to the quadrupolar mode, as we have discussed elsewhere [41], and thus such a mixed phase is additionally stabilized. Finally, the crystal field excitation energy $E_{z}$ cannot be considered to be a constant parameter, as it gets renormalized downwards with increasing doping [39]. Thus, a competition between a ferrodistortive (orthorhombic) and antiferrodistortive tendency occurs and could lead to superstructures with a larger period. In fact, the superstructures observed in oxygen deficient $\mathrm{YBa}_{2} \mathrm{Cu}_{3} \mathrm{O}_{7}$ [49] agree well with this expectation. Also, the anomalous dynamics of the apical oxygens proposed to explain EXAFS [50] and neutron scattering data of a variety of high $T_{c}$ cuprates [51] are consistent with this scenario. Thus, we have to conclude that the structural data [49-51] and the polarized X-ray absorption measurements [5, 52] contradict each other and further experiments seem necessary to clarify the importance of triplet holes in the HTS.

\section{High-spin low-spin $t-J$ model for $d^{8}$ configuration ( $\mathrm{La}_{2} \mathrm{NiO}_{4}$ )}

The triplet $t-J$ model presented in the previous section was an example of a $t-J$ model, where the doping leads to maximal spin states. Frequently one encounters the opposite situation and the spin states $s$ induced by doping are lower than those of the undoped background $S$. In the extreme case this may give localized holes, if the hole has a spin lower than $S-1 / 2$, as in CoO $(S=3 / 2, s=0)$ [53]. We want to concentrate here on a situation with $s=S-1 / 2$. The simplest example is of course the standard singlet $t-J$ model. From the present perspective it is trivial as the orbital degrees of freedom do not enter by construction. A qualitatively new situation occurs for $S=1$ and $s=1 / 2$. Unfortunately, it is impossible to derive a generic model and we shall use a physical situation encountered in $\mathrm{La}_{2} \mathrm{NiO}_{4}$. The states of the background are now either triplets, or singlets, depending on the value of the triplet-singlet excitation energy, $E_{S}=J_{H}-E_{z}$. 
The creation operators of the $m$-component of the triplet and of the singlet at site $i$, respectively, may be written as follows:

$$
\begin{aligned}
& B_{i, 1}^{\dagger}=d_{i x \uparrow}^{\dagger} d_{i z \uparrow}^{\dagger}, \quad B_{i, 0}^{\dagger}=\frac{1}{\sqrt{2}}\left(d_{i x \uparrow}^{\dagger} d_{i z \downarrow}^{\dagger}+d_{i x \downarrow}^{\dagger} d_{i z \uparrow}^{\dagger}\right), \quad B_{i,-1}^{\dagger}=d_{i x \downarrow}^{\dagger} d_{i z \downarrow}^{\dagger}, \\
& A_{i}^{\dagger}=d_{i x \uparrow}^{\dagger} d_{i x \downarrow}^{\dagger},
\end{aligned}
$$

using the notation introduced in Sec. 2. By making a perturbative expansion around the localized $d^{8}$ limit we derived the corresponding superexchange Hamiltonian [54]:

$$
\begin{aligned}
H_{J}= & 2 J \sum_{(i j)}\left[\left(S_{i}+(-1)^{\delta \cdot y} \sqrt{\alpha_{1}} T_{i}\right)\left(S_{j}+(-1)^{\delta \cdot y} \sqrt{\alpha_{1}} T_{j}\right)+\alpha_{2} n_{i A} n_{j A}\right] \\
& +E_{S} \sum_{i} n_{i A},
\end{aligned}
$$

where $J=8 t^{2} / 9 U$, and $\alpha_{1}$ and $\alpha_{2}$ are the parameters depending on the geometry of the wave functions forming the bonds. If $\alpha_{0}=3$, as in Fig. $1, \alpha_{1}=\alpha_{0} / 8$ and $\alpha_{2}=1 / 4$. Here $n_{i A}=A_{i}^{\dagger} A_{i}$ is the singlet number operator at site $i$ and the spin and pseudospin operators, $S_{i}$ and $T_{i}$, are defined by the triplet and singlet components at site $i$ :

$$
\begin{aligned}
& S_{i}^{+}=\sqrt{2}\left(B_{i, 1}^{\dagger} B_{i, 0}+B_{i, 0}^{\dagger} B_{i,-1}\right), \quad S_{i}^{z}=B_{i, 1}^{\dagger} B_{i, 1}-B_{i,-1}^{\dagger} B_{i,-1}, \\
& T_{i}^{+}=\sqrt{2}\left(B_{i, 1}^{\dagger} A_{i}+A_{i}^{\dagger} B_{i,-1}\right), \quad T_{i}^{z}=-\left(A_{i}^{\dagger} B_{i, 0}+B_{i, 0}^{\dagger} A_{i}\right) .
\end{aligned}
$$

These operators obey a $\mathrm{SO}(4)$ (dynamical) algebra, with the canonical commutation relations for the spins,

$$
\left[S_{i}^{\alpha}, S_{i}^{\beta}\right]=2 \epsilon_{\alpha \beta \gamma} S_{i}^{\gamma}, \quad\left[S_{i}^{\alpha}, T_{i}^{\beta}\right]=2 \epsilon_{\alpha \beta \gamma} T_{i}^{\gamma}, \quad\left[T_{i}^{\alpha}, T_{i}^{\beta}\right]=2 \epsilon_{\alpha \beta \gamma} S_{i}^{\gamma} .
$$

The $\mathrm{SO}(4)$ symmetry is explicitly broken by the ligand fields and fully symmetric Hamiltonian is obtained only for $(-1)^{\delta \cdot y} \sqrt{\alpha_{1}} \rightarrow 1$, and $E_{S}, \alpha_{2} \rightarrow 0$. Although the physical origin of our Hamiltonian is completely different, its mathematical structure appears to be conceptually similar to the so-called singlet-triplet models which were studied for the rare earth systems in the sixties [55]. While in the rare earths the spin-orbit coupling dominates and the resulting total angular momenta, $\boldsymbol{J}_{i}$, couple by (dipolar) interactions, the orbital momentum is quenched in our case, and the states with different total spin $S$ interact via kinetic exchange.

As in Sec. 1, we use a classical spin approximation in order to get a qualitative phase diagram of the model (19). Consequently, the ground state at half-filling is written as

$$
\left|\Phi_{0}\right\rangle=\prod_{i}\left[b_{i, 1} B_{i, 1}^{\dagger}+b_{i, 0} B_{i, 0}^{\dagger}+b_{i,-1} B_{i,-1}^{\dagger}+a_{i} A_{i}^{\dagger}\right]|0\rangle,
$$

where the Schwinger bosons $b_{i, m}$ 's are locally parametrized by two angles, $\theta_{i}$ and $\phi_{i}, b_{1, i}=b_{i} \exp \left(-\mathrm{i} \phi_{i}\right) \cos ^{2}\left(\theta_{i} / 2\right), b_{0, i}=b_{i} \sqrt{2} \sin \left(\theta_{i} / 2\right) \cos \left(\theta_{i} / 2\right)$, and $b_{-1, i}=$ $b_{i} \exp \left(\mathrm{i} \phi_{i}\right) \sin ^{2}\left(\theta_{i} / 2\right)$. The two Schwinger bosons, $a_{i}$ and $b_{i}$, satisfy the local constraint, $a_{i}^{2}+b_{i}^{2}=1$. Making a choice of $\phi_{i}=0$, we find the classical energy on a square lattice

$$
\mathcal{E}_{\mathrm{J}}=2 J \sum_{i \delta}\left[b_{i} b_{i+\delta}\left(b_{i} b_{i+\delta}+2 \alpha_{1} a_{i} a_{i+\delta}\right) \cos \left(\Theta_{i \delta}\right)+\alpha_{2} a_{i}^{2} a_{i+\delta}^{2}\right]+E_{S} \sum_{i} a_{i}^{2},
$$


where $\Theta_{i \delta}=\theta_{i}-\theta_{i+\delta}$. While the average boson fields $a$ and $b$ take uniform values at these saddle points, a staggered order parameter for the $z$-th component of both spins $S_{i}$ and pseudospins $\boldsymbol{T}_{i}$ is found. There are three saddle points: the high-spin $\left(b^{2}=1\right)$ and low-spin $\left(a^{2}=1\right)$ phases with energies $\mathcal{E}_{J}(H S)=-z J$ and $\mathcal{E}_{J}(L S)=z J \alpha_{2}+E_{S}$, respectively, and a third saddle point which corresponds to a mixed phase, with $a \neq 0$ and $b \neq 0$. The region of existence of this mixed phase is determined by the physical condition for the amplitude, $0 \leq a^{2} \leq 1$. As shown in Fig. 3, such a mixed state is favoured if $2 \alpha_{1}+\alpha_{2}>1$. For $\alpha_{0}=3$, as for $d_{x^{2}-y^{2}}$ and $d_{3 z^{2}-1}$ orbitals, $2 \alpha_{1}+\alpha_{2}=1$ and it does not exist in the present approximation. In fact, as we will show elsewhere [54], a mixed state is stabilized

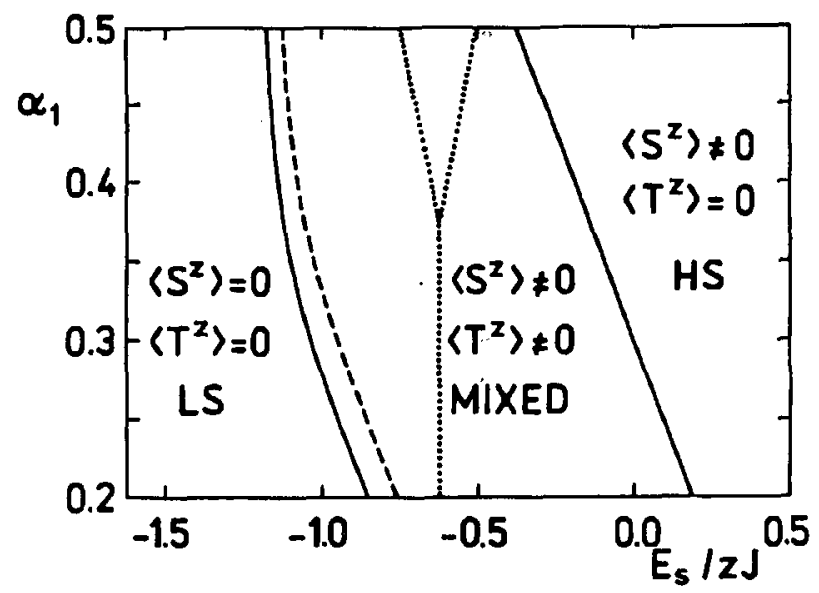

Fig. 3. Phase diagram of the $d^{8} t-J$ model (19) and (26), obtained in the semiclassical spin approximation, as a function of low-spin exciton energy $E_{S} / z J$, and the superexchange interaction $\alpha_{1}$ between the pseudospins $T_{i}$, for $t / z J=1$ and $\delta=0.25$ (full lines). Dashed line marks the transition from the low-spin to high-spin phase. For undoped system $(\delta=0)$ a small region of the mixed phase for $\alpha>3 / 8$ and $E_{S} / z J \approx-5 / 8$ is found (dotted lines).

by quantum fluctuations which are not included in the ansatz (22). The energy gain in this latter mixed phase follows from the mixing of the singlet and triplet configurations such that $\left\langle T_{i}^{z}\right\rangle \neq 0$, while the spin order vanishes, $\left\langle S_{i}^{z}\right\rangle=0$.

Let us now consider the system doped by holes, as in the $\mathrm{NiO}_{2}$ planes of $\mathrm{La}_{2-x} \mathrm{Sr}_{x} \mathrm{NiO}_{4}$. Doping creates low spin states, like $|\sigma\rangle \equiv|x \uparrow x \downarrow z \sigma\rangle$. The hopping of these holes is more complex than that of the triplet holes (12) in Sec. 2 since we want to include the possibility of local creation (annihilation) of the low-spin states $A_{i}$ in the background. By collecting all the possibilities of the hopping we derived [43] the following hopping Hamiltonian expressed in terms of the $B_{i m}$ and $A_{i}$ operators (17), (18), and auxiliary fermions representing the carrier $h_{i \sigma}^{\dagger}$ :

$$
H_{t}=t \sum_{i \delta}\left[X_{i+\delta,+}^{\dagger} h_{i, \uparrow}^{\dagger} h_{i+\delta, \uparrow} X_{i,+}+X_{i+\delta,-}^{\dagger} h_{i, \downarrow}^{\dagger} h_{i+\delta, \downarrow} X_{i,-}\right.
$$


with

$$
\begin{aligned}
& +\left(X_{i+\delta,-}^{\dagger} B_{i, 1}+B_{i+\delta,-1}^{\dagger} X_{i,+}\right) h_{i \uparrow}^{\dagger} h_{i+\delta, \downarrow}+\left(X_{i+\delta,+}^{\dagger} B_{i,-1}+B_{i+\delta, 1}^{\dagger} X_{i,-}\right) h_{i \downarrow}^{\dagger} h_{i+\delta, \uparrow} \\
& \left.+B_{i+\delta, 1}^{\dagger} B_{i, 1} h_{i, \uparrow}^{\dagger} h_{i+\delta, \uparrow}+B_{i+\delta,-1}^{\dagger} B_{i,-1} h_{i, \downarrow}^{\dagger} h_{i+\delta, \downarrow}\right]
\end{aligned}
$$

$$
X_{i, \pm}^{\dagger}=\frac{B_{i, 0}^{\dagger}}{\sqrt{2}} \pm \frac{1}{\sqrt{\alpha_{0}}}(-1)^{\delta \cdot y} A_{i}^{\dagger} .
$$

Equations (19) and (24) define the $t-J$ model for non-maximal spins in the present situation, $H_{t J}=H_{t}+H_{J}$. Looking at this complex Hamiltonian one identifies interesting physical properties of the low-spin hole. Unlike in the triplet $t-J$ model, given by Eqs. (9) and (12), there is no free motion and the hole can only delocalize by exciting spin-flips. Furthermore, the hopping in the low-spin background is hindered because the hole can only delocalize by the hopping of its "wrong" $\left(d_{3 z^{2}-1}\right)$ orbital component, in analogy with the " $x$ " phase of the $d^{9}$ triplet-hole problem. There are also new processes which excite low-spin excitons in a high-spin background, or vice versa. These latter processes are analogous to the "polarization hops" found before in the $d^{9}$ problem. There is, however, one qualitative difference to the singlet $t-J$ model: a moving carrier may either create spin-flips $\left(B_{i, 0}^{\dagger}\right.$ states) in the high-spin phase (usual strings), or it may create more exotic strings of singlet excitons. Such excitations require only relatively low energy in the neighbourhood of the low-spin high-spin transition, and one expects therefore that the singlet states will be partially populated in doped systems. Altogether, the motion of a single hole is more complicated than in the triplet $t-J$ model, not to speak about a finite concentration of doped holes. We will use again the semiclassical approximation to get a qualitative insight in the phases which might occur in a doped compound.

As in the singlet $t-J$ model (2), the crucial step is the representation of the hopping processes by the Schwinger bosons $a_{i}$ and $b_{i}$ and the auxiliary fermions, $h_{i}$. Using again the parametrization of boson fields with $\phi_{i}=0$, and transforming the hole wave functions by a canonical transformation we find [43]:

$$
\begin{aligned}
H_{t}= & t \sum_{i \delta}\left\{\operatorname { c o s } ( \Theta _ { i \delta } ) \left[b_{i+\delta}^{\dagger} b_{i} h_{i, 1}^{\dagger} h_{i+\delta, 1}+\frac{a_{i+\delta}^{\dagger} a_{i}}{\alpha_{0}}\left(h_{i, 1}^{\dagger} h_{i+\delta, 1}+h_{i, 2}^{\dagger} h_{i+\delta, 2}\right)\right.\right. \\
& \left.-\frac{(-1) \delta \cdot y}{\sqrt{\alpha_{0}}}\left(a_{i+\delta}^{\dagger} b_{i} h_{i, 1}^{\dagger} h_{i+\delta, 2}+b_{i+\delta}^{\dagger} a_{i} h_{i, 2}^{\dagger} h_{i+\delta, 1}\right)\right]-\sin \left(\Theta_{i \delta}\right) \\
\times & {\left[\frac{(-1) \delta \cdot y}{\sqrt{\alpha_{0}}}\left(a_{i+\delta}^{\dagger} b_{i}-b_{i+\delta}^{\dagger} a_{i}\right) h_{i, 1}^{\dagger} h_{i+\delta, 1}+\frac{a_{i+\delta}^{\dagger} a_{i}}{\alpha_{0}}\left(h_{i, 1}^{\dagger} h_{i+\delta, 2}-h_{i, 2}^{\dagger} h_{i+\delta, 1}\right]\right\} . }
\end{aligned}
$$

The meaning of the different hoppings becomes clear in the semiclassical limit. It is well known from the $t-J$ model that the competition between the FM tendency coming from the hole hopping and the AF exchange at half-filling gives rise to either uniform canting of the spins [56], or to a spiral spin structure [57]. In the low-spin phase, on the contrary, holes with both spin directions can hop and 
thus both "1" and "2" quasiparticle states appear in the terms $\propto a^{2}$ in Eq. (26). The terms which describe the hopping in the mixed phase, $\propto a b$, are novel. They come in two varieties: (i) the ones $\propto \cos \left(\Theta_{i \delta}\right)$, describing the mixing of the two hole sectors, and (ii) the terms at maximum in the Néel state $\left(\alpha \sin \left(\Theta_{i \delta}\right)\right)$, representing the propagating "high-spin" holes. This form of propagation is new and does not suppress the magnetic order. To get more insight into the phase diagram of the model, one has to consider either uniform ferro-orbital (FO), or staggered antiferro-orbital (AFO) phasing of "orbital" variables, $a_{i}$ and $b_{i}$, on the square lattice. Thus, we have diagonalized the hopping Hamiltonian (26) for the canted and spiral configurations of the spin, both characterized by a single parameter $\delta \theta$ which stands for the rotation of a pair of spins with respect to the Néel state. As we show elsewhere [43], one finds at small doping $(t \delta \ll 2 J)$ for the classical energy

$$
\mathcal{E}_{t J}=-z J b^{2}\left(b^{2}-2 \alpha_{1} a^{2}\right) \cos (\delta \theta)+z J \alpha_{2} a^{4}+E_{S} a^{2}+\delta \omega_{1}
$$

where $\omega_{1}$ is the minimum over $k$ of the lowest eigenvalue $\omega_{1}(k)$ found from the diagonalization of $H_{t}$, that the AFO is more stable than the FO order. The numerical results are summarized in Figs. 3 and 4. For positive low-spin exciton energies

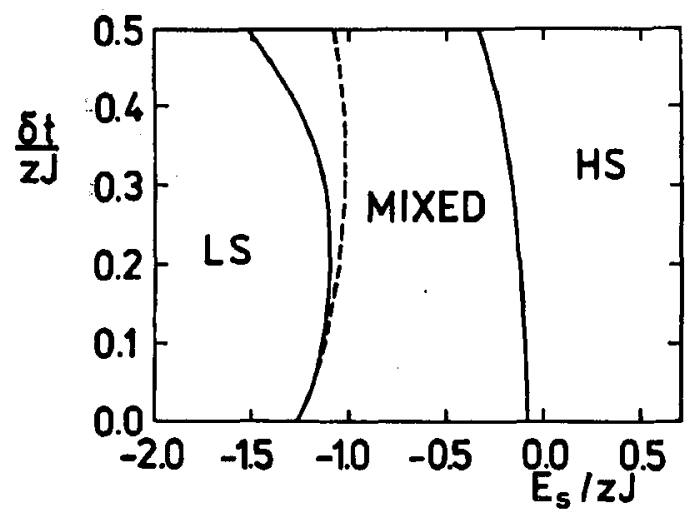

Fig. 4. Phase diagram of the $d^{8} t-J$ model (19) and (26), obtained in the semiclassical spin approximation, as a function of low-spin exciton energy $E_{S} / z J$, and doping $t \delta / z J$ (full lines). Dashed line marks the transition from the low-spin to high-spin phase.

$\left(E_{S}>0\right)$ we find the conventional high-spin phase to be stable. It develops a twist in the spin order, with $\sin (\delta \theta)=t \delta / 2 J$, and we cannot distinguish between a spiral or canted structure in the classical approximation [20]. However, we expect that quantum fluctuations will stabilize a spiral ordering, similar as in the $t-J$ model [25]. The FM phase is stable at large dopings, $t \delta_{\mathrm{c}}=z J$. If $E_{S} \ll 0$, the low-spin phase is stable, while the holes move freely. By comparison the energies of the high-spin and low-spin phases we find that the transition line is

$$
\frac{E_{S}}{z J}=-\left(1+\alpha_{2}\right)+\frac{1}{\alpha_{0}}\left(\frac{t \delta}{J}\right)-\frac{1}{8}\left(\frac{t \delta}{J}\right)^{2} .
$$

In the vicinity of this transition we find a mixed phase, separated by first-order boundaries from the other phases, with the doped holes having momenta near 
$k=(0, \pi)$ (see Fig. 3). The mixed phase is characterized by no tendency to spiral in the spin component, but instead by a staggered (AF) spin order $\left\langle S_{i}^{z}\right\rangle$ with reduced amplitude, and an AFO ordering in the orbital $(a, b)$ channel. The pseudospins $\boldsymbol{T}_{i}$ have a net FM orientation because the spin and orbital staggerings cancel each other. We note that the mixed phase exists in a rather broad range of $E_{S} / z J$, regardless of the actual ratio of the hopping elements $\alpha_{0}$, or the superexchange interaction between pseudospins $\sim \alpha_{1}$.

Different from the transition between the high-spin and the mixed phase, the transition from the mixed to the low-spin phase depends on the doping level. For larger doping the mixed phase expands at the expense of the low-spin phase. Both transitions to the high-spin and low-spin phases are first order. The origin of this first-order behaviour is similar to what we found in Sec. 2. There is a competition originating in the kinetic energy because the doped holes occupy different regions in $k$-space: the hole has a momentum close to $(\pi / 2, \pi / 2)$ in the spiral phase and to $(0,0)$ in the low-spin phase, respectively, while in the mixed phase it has a momentum close to $(0, \pi)$. In spite of the first order nature of the phase transitions, we do not find a tendency towards phase separation in the present case.

In Fig. 4 we present the phase diagram of the $t-J$ model as a function of doping. The stability of the phases is determined by the universal ratio $t \delta / z J$. Obviously, this classical phase diagram is merely suggestive. The largest corrections are expected for the transition line between the high-spin spiral phase and the mixed phase, because the energy of the former phase is severely underestimated in this classical theory. The role of quantum corrections in the mixed state is less clear. Although the excitations are massive, we already noticed in Sec. 2 that the used classical approximation is possibly not very accurate at the smallest length scales [57].

\section{Summary and outlook}

The presented material demonstrates that the motion of holes in the MottHubbard insulators is highly nontrivial and despite of recent progress, remains to be fully understood. In any case, this concerns the complex situations, where orbital degrees of freedom start to play a role. As we have shown, depending on the physical situation, there are different $t-J$ models describing the strongly correlated doped materials. Of course, it would be quite disappointing, if this physics would be compound-dependent and no general statements could be made. Fortunately, this is not the case. Although, the phase diagrams do depend on the underlying microscopies, there are certain definite trends observed in all of these cases. First of all, the competition between the magnetic, $\sim J$, and kinetic $\sim t$, energy, well known from the $t-J$ model [30], could be identified as well in the more complicated models presented in this paper. Consequently, the doped systems exhibit tendencies towards incommensurate spin structures, like spirals or canting. Such structures, although found on the mean-field level, may be unstable in reality (at least at small doping) and the system may phase separate into hole-poor and hole-rich regions. Secondly, the presence of the orbital degrees of freedom adds a new energy scale to the problem which makes it already interesting without doping. The competition between the magnetic and orbital (excitonic) energy may stabilize new magnetic phases, as we have shown for the $d^{9}$ spin-orbital model [37]. The 
regions of stability of these new phases are, however, quite limited and so far only one example of a system to which such a model applies $\left(\mathrm{K}_{2} \mathrm{CuF}_{4}[45]\right)$ has been found.

Hole dynamics in the spin-orbital models is very interesting. A moving hole may not only destroy the magnetic order, as in the $t-J$ model, but it may also compete with the orbital ordering. As a consequence, the hole dresses by magnetic, as well as by orbital excitations and new phases occur, where it is dressed by both. Such new phases were found both in the spinless model simulating the situation in the high-spin (triplet hole) $t-J$ model in $d^{9}$, and in the low-spin $(s=1 / 2) t-J$ model in $d^{8}$. Therefore, we believe that the binding of excitons to moving holes, as observed in these phases, is rather a rule than an exception in the Mott-Hubbard insulators. This may be of importance in the cuprates and in the nickelates. For instance, we believe that local excitonic excitations might be responsible for the virtual disappearance of the Mott-Hubbard gap from the inverse photoemission of doped nickelates, as suggested by detailed calculations by van Elp et al. [58]. Furthermore, the excitons induced by doping have a tendency to couple to phonons. For instance, the quadrupolar distortions are promoted by the mixed orbital order of $\mathrm{K}_{2} \mathrm{CuF}_{4}$. Finally, it was shown recently that the spectral function of a single hole is drastically modified by modest electron-phonon interactions and the dressed holes become heavier [59]. Most probably, the ground states of certain Mott-Hubbard insulators cannot be understood solely in terms of the electronic models.

By considering the orbital degrees of freedom, we have derived a new variety of $t-J$ models. One may wonder whether a single moving hole alters the ground state in a way qualitatively similar to that known from the standard $t-J$ model. We know already one situation (the triplet hole $t-J$ model) where this is not the case: the hole dresses itself with spin waves, but the vertex diverges at small momenta and the quasiparticle picture breaks down [46]. It remains to be investigated how the hole moves in the low-spin $t-J$ model derived for $d^{8}$ configuration.

One could argue that it is not clear whether the physics described by the $t-J$ model is ever realized. In fact, we know already one example, where the $t-J$ model is useful and allows us to understand the angle resolved photoemission spectra of $\mathrm{NiO}$ [60]. Taking into account a realistic electronic structure, one may derive a model Hamiltonian of $t-J$ variety which describes the hole within one of the two oxygen subbands, interacting with the spin waves propagating in the $\mathrm{AF}$ background. More details are given in a separate publication [60]; here we want to state only the main result of this study. In self-consistent Born order we find the spectral functions composed of coherent quasiparticle modes, being bound states of the Zhang-Rice type, and, in addition, of an incoherent background. The main features of the obtained spectra agree quite well with the experimentally found dispersions [61]. Thus, the experimental spectra can be interpreted in this case as supporting the complex, strongly-correlated, character of the electronic states in $\mathrm{NiO}$, with both itinerant and localized aspects appearing in the spectra, rather than supporting the naive picture emerging from the band structure calculations.

Summarizing, we would like to emphasize that only few universal concepts apply to the whole family of $t-J$ models. These are: (i) the competition between magnetic and kinetic energies, as well as (ii) the binding of excitons to the moving 
holes. The $t-J$ model itself proved to be very useful in developing the theoretical concepts in this field. As it stands, however, it is too oversimplified to explain the physics of doped Mott-Hubbard insulators at large. For instance, in its application to the HTS, the hole spectral functions obtained from this model [13, 22] do reproduce only the low-energy part of the spectra obtained either by the exact diagonalization of $\mathrm{Cu}_{4} \mathrm{O}_{12}$ cluster [62], or from the strong-coupling analytic treatment of the Emery model [63]. Thus, they have been successfully applied to the optical properties of HTS [64], but cannot explain the photoemission spectra. We are convinced that further effort to understand the properties of the complex $t-J$ models related to particular physical situations will result in better and more quantitative understanding of the physical phenomena in doped transition metal oxides, the best characterized examples of the Mott-Hubbard insulators in nature.

\section{Acknowledgments}

We thank L.F. Feiner, P. Horsch, P.B. Littlewood, the late S. Schmitt-Rink, G.A. Sawatzky, and J. Spalek for valuable discussions which contributed to our deeper understanding of the subject. A.M.O. acknowledges the kind hospitality of M.C. Desjonquères, and D. Spanjaard at Service de Recherche sur les Surfaces et l'Irradiation de la Matière, CE Saclay, and at Laboratoire de Physique des Solides, Université Paris-Sud, Orsay, where this study was completed, as well as the partial support by the Committee for Scientific Research, project No. 203869101.

\section{References}

[1] D. Vollhardt, Rev. Mod. Phys. 56, 99 (1984).

[2] F. Marsiglio, J.E. Hirsch, Phys. Rev. B 41, 6435 (1990): J.E. Hirsch, Phys. Rev. B 40, 2354 (1989); ibid. 41, 6820 (1990); F. Marsiglio, J.E. Hirsch, Phys. Rev. B 41, 6435 (1990).

[3] C.T. Chen, L.H. Tjeng, J. Kwo, H.L. Kao, P. Rudolf, F. Sette, R.M. Fleming, Phys. Rev. Lett. 68, 2543 (1992); E. Pellegrin, N. Nücker, J. Fink, S.L. Molodtsov, A. Gutiérrez, E. Navas, O. Strebel, Z. Hu, M. Domke, G. Kaindl, S. Uchida, Y. Nakamura, J. Markl, M. Klauda, G. Saemann-Ischenko, A. Krol, J.L. Peng, Z.Y. Li, R.L. Greene, Phys. Rev. B 47, 3354 (1993).

[4] J. Hubbard, Proc. R. Soc. Lond. A 276, 238 (1963).

[5] C.M. Varma, S. Schmitt-Rink, E. Abrahams, Solid State Commun. 62, 681 (1987); V.J. Emery, Phys. Rev. Lett. 58, 2794 (1987).

[6] N.E. Bickers, D.L. Cox, J.W. Wilkins, Phys. Rev. B 36, 2036 (1987); O. Gunnarsson, K. Schönhammer, Phys. Rev. B 28, 4315 (1983).

[7] D.L. Cox, Phys. Rev. Lett. 59, 1240 (1987).

[8] M. Cyrot, C. Lyon-Caen, J. Phys. (Paris) 36, 253 (1975); C. Lacroix, J. Phys. C 13, 5125 (1980).

[9] P. Fulde, J. Phys. F 18, 601 (1988); A.C. Hewson, The Kondo Problem to Heavy Fermions, Cambridge University Press, Cambridge 1992.

[10] Y. Nagaoka, Phys. Rev. 147, 392 (1966).

[11] W. von der Linden, D.M. Edwards, J. Phys., Condens. Matter 3, 4917 (1991). 
[12] K.A. Chao, J. Spałek, A.M. Oleś, J. Phys. C 10, L271 (1977).

[13] K.J. von Szczepanski, P. Horsch, W. Stephan, M. Ziegler, Phys. Rev. B 41, 2017 (1990).

[14] P.W. Anderson, Science 256, 1526 (1992).

[15] F.C. Zhang, T.M. Rice, Phys. Rev. B 37, 3759 (1988).

[16] P.A. Bares, G. Blatter, M. Ogata, Phys. Rev. B 44, 130 (1991).

[17] E. Manousakis, Rev. Mod. Phys. 63, 1 (1991).

[18] W.F. Brinkman, T.M. Rice, Phys. Rev. B 2, 1324 (1970).

[19] L.N. Bulaevskii, E.L. Nagaeev, D.L. Khomskii, Zh. Exp. Teor. Fiz. 54, 1562 (1968); [Sov. Phys. JETP 27, 836 (1968)]; B.I. Shraimann, E.D. Siggia, Phys. Rev. Lett. 60, 740 (1988).

[20] S.A. Trugman, Phys. Rev. B 37, 1597 (1988).

[21] S. Schmitt-Rink, C.M. Varma, A.E. Ruckenstein, Phys. Rev. Lett. 60, 2793 (1988); C.L. Kane, P.A. Lee, N. Read, Phys. Rev. B 39, 6880 (1989).

[22] E. Dagotto, A. Moreo, R. Joynt, S. Bacci, E. Gagliano, Phys. Rev. B 41, 2585 (1990); D. Poilblanc, T. Ziman, H.J. Schulz, E. Dagotto, Phys. Rev. B 47, 14267 (1993).

[23] A.M. Oleś, Int. J. Mod. Phys. B 3, 1789 (1989); R. Eder, K.W. Becker, Phys. Rev. $B$ 44, 6982 (1991).

[24] G. Martínez, P. Horsch, Phys. Rev. B 44, 317 (1991).

[25] C.L. Kane, P.A. Lee, T.K. Ng, B. Chakraborty, N. Read, Phys. Rev. B 41, 2653 (1990).

[26] C. Jayaprakash, H.R. Krishnamurthy, S. Sarker, Phys. Rev. B 40, 2610 (1989).

[27] H. Eskes, L.F. Feiner, G.A. Sawatzky, Physica C 160, 424 (1989).

[28] A. Auerbach, B.E. Larson, Phys. Rev. B 43, 7800 (1991).

[29] V.J. Emery, S.A. Kivelson, H.Q. Lin, Phys. Rev. Lett. 64, 475 (1990).

[30] V.J. Emery, S.A. Kivelson, Physica C 209, 597 (1993).

[31] H.J. Schulz, Phys. Rev. Lett. 64, 1445 (1990); D. Poilblanc, T.M. Rice, Phys. Rev. $B$ 39, 9749 (1989).

[32] J. Zaanen, O. Gunnarsson, Phys. Rev. B 43, 7800 (1991).

[33] K. Yosemitsu, A.R. Bishop, J. Lorenzana, Phys. Rev. Lett. 69, 2906 (1992).

[34] K. Yosemitsu, A.R. Bishop, J. Lorenzana, Phys. Rev. B 47, 8065 (1993); ibid. 47, 12059 (1993).

[35] K.C. Haas, in: Solid State Physics, Vol. 42, Eds. H. Ehrenreich, D. Turnbull, Academic Press, Orlando 1989, p. 213.

[36] A.M. Oleś, Phys. Rev. B 28, 327 (1983).

[37] J.C. Slater, G.F. Koster, Phys. Rev. 94, 1498 (1954).

[38] J.B. Grant, A.K. McMahan, Phys. Rev. B 46, 3440 (1992).

[39] D.I. Khomskii, Physica B 171, 44 (1991).

[40] J.B. Grant, A.K. McMahan, Phys. Rev. Lett. 66, 488 (1991).

[41] J. Zaanen, A.M. Oles', L.F. Feiner, in: Dynamics of Magnetic Fluctuations in High Temperature Superconductors, Eds. G. Reiter, P. Horsch, G.C. Psaltakis, Plenum Press, New York 1991, p. 241. 
[42] A.M. Oles, J. Zaanen, Acta Phys. Pol. B 24, 825 (1993).

[43] J. Zaanen, A.M. Oles', Phys. Rev. B 48, in press (1993).

[14] K.I. Kugel, D.I. Khomskii, Solid State Commun. 13, 763 (1973).

[45] Y. Ito, J. Akimutsu, J. Phys. Soc. Jpn. 33, 1333 (1976); L.D. Khoi, P. Veillet, Phys. Rev. B 11, 4128 (1975); W. Kleemann, Y. Farge, J. Phys. (Paris) 36, 1293 (1975).

[46] J. Zaanen, A.M. Oleś, Phys. Rev. B 48, 7197 (1993).

[47] W. Weber, Z. Phys. B 70, 323 (1988).

[48] M. Jarrell, H.R. Krishnamurthy, D.L. Cox, Phys. Rev. B 38, 4584 (1988); D.L. Cox, M. Jarrell, C. Jayaprakash, H.R. Krishnamurthy, J. Deisz, Phys. Rev. Lett. 62, 2188 (1989).

[49] T. Krekels, T.S. Shi, J. Reyes-Gasga, G. van Tendeloo, J. van Landuyt, S. Amelinckx, Physica C 167, 677 (1990).

[50] A.R. Bishop, J. Röhler, A. Larisch, in: Electronic Properties of High-Tc Superconductors and Related Compounds, Eds. H. Kuzmany, M. Mehring, J. Fink, Springer Verlag, Berlin 1990.

[51] T. Egami, B.H. Toby, S.J. Bilinge, C. Janot, J.D. Jorgensen, D.G. Hinks, M.A. Subramanian, M.K. Crawford, W.E. Farneth, E.M. McCarron, in: Electronic Structure and Mechanisms of High- $T_{c}$ Superconductivity, Eds. J. Ashkenazi, G. Vezzoli, Plenum Press, New York 1991.

[52] C. Di Castro, L.F. Feiner, M. Grilli, Phys. Rev. Lett. 66, 3209 (1991).

[53] J. van Elp, J.L. Wieland, H. Eskes, P. Kuiper, G.A. Sawatzky, F.M.F. de Groot, T.S. Turner, Phys. Rev. B 44, 6090 (1991).

[54] L.F. Feiner, A.M. Oleś, J. Zaanen, unpublished.

[55] P. Fulde, I. Peschel, Adv. Phys. 21, 1 (1972); Y.Y. Hsieh, M. Blume, Phys. Rev. B 6, 2684 (1972).

[56] P.W. Anderson, H. Hasegawa, Phys. Rev. 100, 675 (1955); P.-G. de Gennes, Phys. Rev. 118, 141 (1960).

[57] B.I. Shraiman, E.D. Siggia, Phys. Rev. Lett. 61, 467 (1988); Phys. Rev. B 42, 2485 (1990).

[58] J. van Elp, H. Eskes, P. Kuiper, G.A. Sawatzky, Phys. Rev. B 45, 1612 (1992).

[59] A. Ramšak, P. Horsch, P. Fulde, Phys. Rev. B 46, 14305 (1992).

[60] J. Bała, A.M. Oleś, J. Zaanen, unpublished.

[61] Z.-X. Shen, C.K. Shih, O. Jepsen, W:E. Spicer, I. Lindau, J.W. Allen, Phys. Rev. Lett. 64, 2442 (1990); Z.-X. Shen, R.S. List, D.S. Dessau, B.O. Wells, O. Jepsen, A.J. Arko, R. Barttlet, C.K. Shih, F. Parmigiani, J.C. Huang, P.A.P. Lindberg, Phys. Rev. B 44, 3604 (1991).

[62] W. Stephan, P. Horsch, in: Dynamics of Magnetic Fluctuations in High Temperature Superconductors, Eds. G. Reiter, P. Horsch, G.C. Psaltakis, Plenum Press, New York 1991, p. 175.

[63] P. Unger, P. Fulde, Phys. Rev. B 47, 8947 (1993).

[64] A. Moreo, E. Dagotto, Phys. Rev. B 42, 4786 (1990); I. Sega, P. Prelovšek, Phys. Rev. B 42, 892 (1990); W. Stephan, P. Horsch, Phys. Rev. B 42, 8736 (1990); C.X. Chen, B. Schüttler, Phys. Rev. B 43, 3771 (1991). 